\title{
Designing a Model for Developing Key Human Resource Competencies in the Red Crescent Society of the Islamic Republic of Iran
}

\author{
Homayoun Nouri $^{1,5} \mathbb{D}$, Nour Mohammad Yaghoubi ${ }^{2} \mathbb{D}$, Ebrahim Haddadi $^{3} \mathbb{D}$, Seid Mehdi Vise ${ }^{4} \mathbb{C}$
}

Date of submission: 21 Jan. 2020 Date of acceptance: 15 Nov. 2020

\begin{abstract}
INTRODUCTION: In recent years, competency has emerged as a major issue which plays a significant role in responding to challenges facing organizational management in a rapidly changing environment. In this regard, the competency-based approach puts emphasis on individuals and highlights the importance of human resources to achieve organizational goals. The Red Crescent Society of the Islamic Republic of Iran is a human-centered organization; that is to say, most of the services provided to the target communities rely on the capabilities of human resources. Therefore, the current study aimed to design a model for the development of key human resource competencies in the Red Crescent Society of the Islamic Republic of Iran.

METHODS: This applied qualitative study was conducted based on an exploratory approach and used mixed-type data. Data collection was performed in two qualitative and quantitative phases. The first phase was conducted based on grounded theory, while a quantitative research method was used in the next phase. The statistical population of the study in the qualitative section included managers and human resource experts of the Red Crescent Society, as well as university professors and experts, who were selected using purposive sampling and were interviewed until saturation data (25 interviews). The statistical population of the quantitative section included 8,451 managers and employees of the Red Crescent Society of all provinces of Iran. The sample size was calculated at 361 cases using Krejcie and Morgan sample size determination table.

FINDINGS: In the present study, among the seven principles of the Red Cross and Red Crescent Movement, the five principles of humanity, impartiality, neutrality, independence, and voluntary service were regarded as infrastructure requirements for the development of human resource competencies and were axially coded in causal conditions. Thereafter, the relationship between these principles and human resources competencies in the Red Crescent Society was determined using PLS software.

CONCLUSION: As evidenced by the obtained results, the employment of appropriate strategies for evaluating and developing key human resource competencies resulted in the following consequences: human resource efficiency, provision of desirable social services, community health improvement, enhancement of human values, reinforcement of trust and public participation, and improvement of the financial capacity of the Red Crescent Society. The results indicated that competency development among Red Crescent staff is positively and significantly correlated with the principles of humanity, neutrality, impartiality, and independence.
\end{abstract}

Original Article

Keywords: Competence; Human Resources; Red Crescent Society.

How to cite this article: Nouri H, Yaghoubi NM, Haddadi E, Vise SM. Designing a Model for Developing Key Human Resource Competencies in the Red Crescent Society of the Islamic Republic of Iran. Sci J Rescue Relief 2020; 12(3): 185-196.

1-PhD Student, Department of Public Administration, Faculty of Literature and Humanities, Islamic Azad University, Zahedan Branch, Iran

2-PhD, Department of Public Administration, Faculty of Management and Economics, University of Sistan and Baluchestan, Zahedan, Iran 3-PhD, Department of Public Administration, Faculty of Literature and Humanities, Islamic Azad University, Zahedan Branch, Iran

4-PhD, Department of Public Administration, Faculty of Literature and Humanities, Ilam University, Iran

5- Research Center for Emergency and Disaster Resilience, Red Crescent Society of the Islamic Republic of Iran, Tehran, Iran Correspondence to: Nour Mohammad Yaghoubi, Email: yaghoubi@mgmt.usb.ac.ir 


\section{Introduction}

$\mathrm{O}$ rganizations have turned their focus toward the development and evaluation of managerial competencies since the mid1980s. The persistent belief in the substantial benefits of enhanced managerial competencies for organizations has been the reason behind this assiduous attention (1). The competency-based approach to employees, as a strategic principle, is in fact the link between employees and their organization. Therefore, organizations can compete with their counterparts around the world relying on the competencies of employees (2).

Competency is a common technique for evaluating human resources in different organizations that allow the people in authority to assess and develop the capabilities of employees important to the organization (3). Moreover, it enables the employees to distinguish between the best and worst employees, find and attract suitable employees, and link employees' capabilities to the strategic goals of the organization. The management of employees' competencies should be based on the principles of valid competency management presented through the development of competency models and the use of different methods of competency assessment (4). Therefore, it can be argued that the development of competencies is a factor that helps organizations to successfully deal with changing environments (5).

Based on the aforementioned issues, it can be acknowledged that attention to human resources and their development as the most valuable organizational assets demonstrates that human beings are regarded as a decisive and effective partner in organizations. In addition, the development of human resource competencies leads to the comprehensive and balanced development of organizations. Therefore, the implementation of effective measures in the field of human resource management paves the ground for the expansion of core competencies in the world of business (6).

Competencies are often identified as the knowledge, expertise, skills, and abilities individuals need to perform their job roles and responsibilities. The European Commission (EC) defined competency as "the proven ability to use knowledge, skills and personal, social, and/or methodological abilities, in work or study situations and professional and personal development." Furthermore, the term competency has been defined from various perspectives, such as general competencies, soft skills, business skills, and technical competencies. Consequently, the term competency reflects a functional view of the skills required to perform a particular task (7).

Therefore, due to the crucial importance of human resources competencies, special attention should be devoted to this critical issue in organizations. The Red Crescent Society of the Islamic Republic of Iran is no exception in this regard. So far, no activity has been performed on this field by Red Crescent Society as a relief and non-profit organization. Nevertheless, most activities performed in this society are focused on preparedness and first aid training. Moreover, under the slogan "ready family in disasters", numerous activities in this organization are directed toward public relief education.

Furthermore, the Red Crescent Society, as the custodian of relief and rescue, is responsible for the management of disaster victims. This issue is of increasing importance due to science and technology developments, rapid technological advancement, and the growing population of the world (8). Therefore, the competencies of the Red Crescent aid workers should be promoted so that they can effectively perform their activities in an organization which plays a peculiar role in providing relief to community members in the time of crises.

The current situation of the Iranian Red Crescent Society is indicative of the absence of a comprehensive model for assessing and developing human resources competencies. The Red Crescent Society of the Islamic Republic of Iran is a human-centered organization; that is to say, most of the services provided to target communities rely on the capabilities of human resources. Therefore, the lack of a model for evaluating and developing the competencies of these valuable resources has significant consequences that not only affect human resources but also are reflected in all aspects of their organization.

The managers of this organization are not satisfied with this situation, and the evaluation of managers and employees based on models other than competencies gives rise to considerable dissatisfaction among the whole organization and reduces work efficiency. In addition, it leads to other problems, such as weakness in succession planning. Therefore, the critical importance of the 


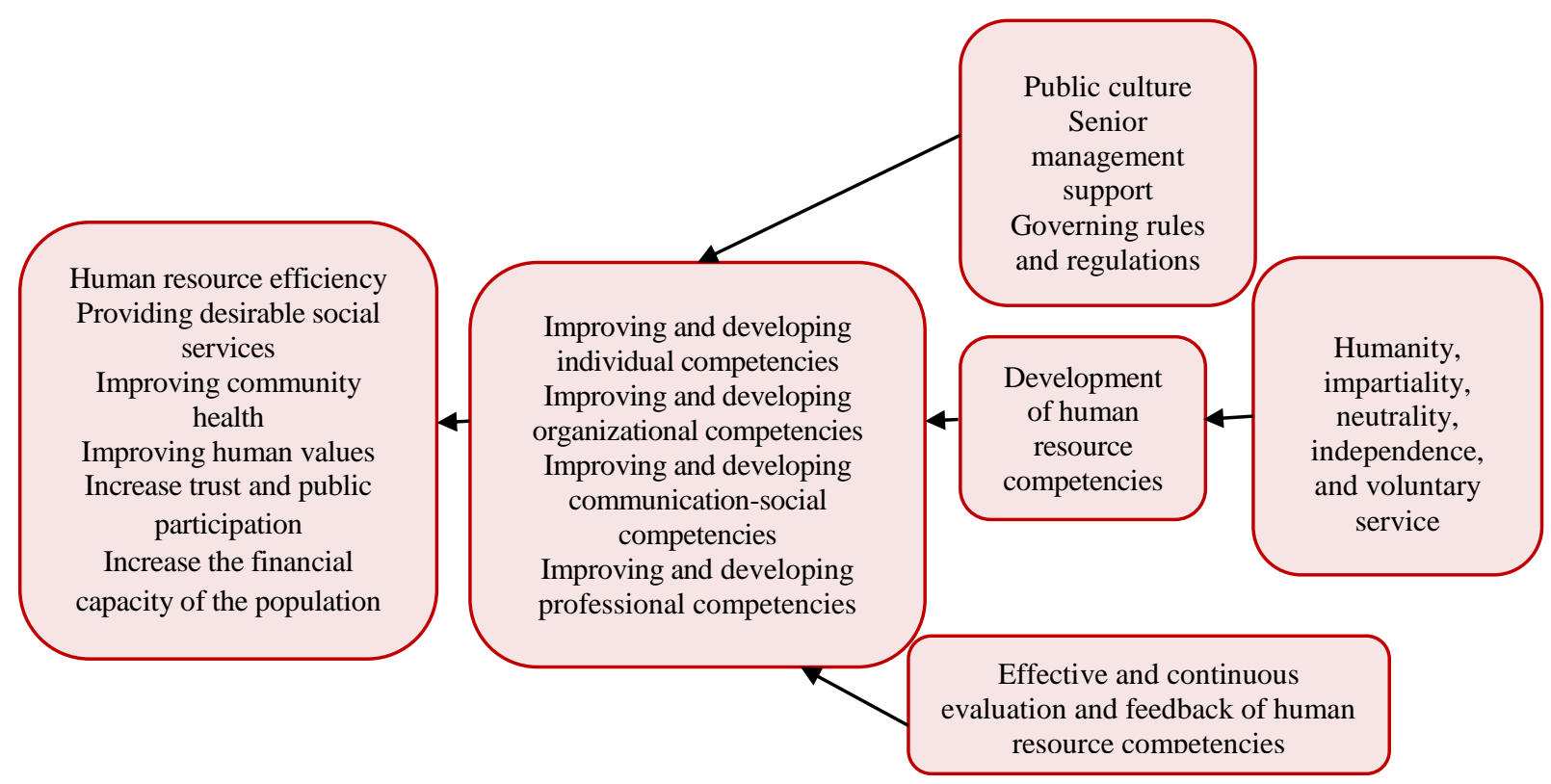

Figure 1. The final model for the development of key human resource competencies in the Iranian Red Crescent Society

evaluation and development of competencies in the Iranian Red Crescent Society and the dearth of studies in this field tempted the researcher to fill this research gap. In this regard, the present study aimed to design a model for the development of key human resource competencies in the Red Crescent Society of the Islamic Republic of Iran (Figure 1).

\section{Competency model}

The competency model is a measurable and observable model of the skills and behaviors needed to successfully perform activities in work environments. The competency model aims to provide a model for integrating the performance of the human resource system for the effective performance of organizations.

\section{Methods}

This applied qualitative study was conducted based on an exploratory approach using Grounded Theory. Grounded theory, as an inductive and exploratory research method, allows researchers in various subject areas to formulate their own theories instead of relying on existing ones. Grounded theory has two approaches proposed by Glasser and Strauss. In the current study, Strauss's objectivist method was used to analyze the data since it is a systematic method. Therefore, it can be stated that this applied qualitative study was conducted based on an exploratory approach and used mixed-type data. Data collection was performed in two qualitative and quantitative phases using interviews and questionnaires.

The main method of data collection was the semi-structured in-depth interview technique, in which the researcher prepares the necessary topics before the scheduled interview. Nonetheless, the questions are open and are not prepared in advance, and the interview process largely relies on questions that spontaneously arise in the interaction between the interviewer and the interviewee. In the present study, data collection reached saturation after 25 interviews with managers and experts in human resource development of Iranian Red Crescent Society, experts, and university professors in the field of management. However, the 26th interview was conducted for much more assurance.

In the quantitative section, information was obtained through questionnaires. Since the present research was based on a hybrid design (qualitative and quantitative), sampling was also performed based on the rules of this method. The statistical population in the qualitative section included the managers and experts of Iranian Red Crescent Society, as well as experts and academics in the field of management, and supervisors and consultants. Snowball sampling was performed in the qualitative part of the research (interview). In this method, firstly, one of the managers, university professors, and management experts is interviewed. Thereafter, that interviewee will be 
asked to introduce friends, acquaintances, or people who may be suitable for the assessment. In the quantitative section, the statistical population of this study included 8451managers and employees of the Red Crescent Society of all provinces in Iran.

The sample sized was calculated at 361 cases using Krejcie and Morgan's sample size determination table and proportionate stratified random sampling method. The categories of this research were all the provinces of Iran, and a number of managers and experts in the field of human resource development were randomly selected from each province and were entered into the study. Among the qualitative and interpretive methods, the grounded theory which offers major benefits to researchers was used to analyze the qualitative data. This method has a high capacity to interpret complex phenomena and assess and describe phenomena in their natural context.

There is a theoretical gap in the subject under study (i.e., the development of human resource competencies in the Red Crescent Society). In other words, there is no specific theory in this field, especially the human resources of the Red Crescent Society with the unique characteristics of this organization. Therefore, the research team decided to collect data through interviewing experts in this organization and designing a model by inductive content analysis and coding of transcriptions. Thereafter, the designed model was validated using the grounded theory. Therefore, in the grounded theory, data analysis is performed by coding interviews and textual data. Information coding consists of three steps: open coding, axial coding, and selective coding

In open coding, by referring to the initial codes extracted from the interviews (coding of key points), the codes that referred to a common topic were grouped and constructed concepts, and categories emerged from the comparison and classification of concepts. A total of 72 concepts, 568 sub-concepts, and 19 categories were identified. MAXQDA software (version 10) was used to organize qualitative data. In the axial coding stage, in order to link the main categories to subcategories, a paradigm model was used to determine the causal conditions, axial phenomenon, contextual factors, intervening variables, strategies, and consequences that are the components of the axial coding. Subsequently, the categories were related, and the relevant system was presented through selective coding and based on the identified relationship between categories and subcategories in open and axial coding.

Descriptive and inferential statistics were used to analyze the quantitative data. Descriptive statistical methods, relative frequency distribution table, and percentage charts were used in the descriptive part of the research. On the other hand, structural equation modeling was used in the inferential statistical analysis.

\section{Findings}

\section{Describing the studied variables and interview questions}

To obtain the data that could meet the objectives of the research, in-depth semistructured interviews were conducted with professors, managers, and management experts. Open coding is performed in two stages of primary and secondary coding. Primary coding can be conducted by line-by-line, phrase-byphrase, or paragraph-by-paragraph coding of data. A concept or code is attached to each of them. In secondary coding, after concept extraction, similar and common codes are organized in a single category by comparing concepts. Therefore, a mass of data (codes-concepts) is reduced to a certain and limited number of major categories.

\section{Open coding}

In open coding, the researcher extracted the specific concepts and coded them by line-by-line revision of interviews using MAXQDA qualitative data analysis software (version 10). A total of 72 different codes or concepts which were repeated 568 times in the interviews were extracted in the first stage. In Table 1, an attempt was made to replace similar and common concepts with a single category and identify the main categories of research (Table 1).

\section{Axial coding}

In axial coding, the researcher selects a core category from the open coding stage and positions it at the center of study (as a central phenomenon or category); thereafter, other categories are related to it. According to the main topic of the interview and the subject under study, the central phenomenon of this research was the development of key human resource competencies in the Iranian Red Crescent Society. In the meantime, 
causal conditions are categories that affect the central phenomenon.

In the present study, among the seven principles of the Red Cross and Red Crescent Movement, the five principles of humanity, impartiality, neutrality, independence, and voluntary service were regarded as infrastructure requirements for the development of human resource competencies and were axially coded in causal conditions. In other words, the development of key human resource competencies is one of the basic requirements for achieving these five principles. The improvement and development of individual competencies, improvement, and development of organizational competencies, improvement and development of social

Table 1. Categorization of codes

Category

Individual competencies

Communication-social competencies

Professional competencies

Organizational competencies

Human resource efficiency

Provision of desirable social services

Community health improvement

Improving human values

Increasing the financial capacity of the Society

Increasing trust and public participation

Principle of humanity

Principle of voluntary service

Impartiality

Neutrality

Independence

Public culture

Senior management support

Governing rules and regulations

Effective and ongoing evaluation and feedback on human resource competencies

\section{Related concepts}

Responsibility / Discipline / Values and Attitudes / Personality / Ethics and Literature / Speed of Action / Intelligence and Talent / Self-Confidence / Patience

Appropriate workplace communication / Ability to participate and work in a team / Active listening / Establishing emotional communication with peers / Ability to transfer experiences and skills to others / Creating a sense of friendship and cooperation between people / Effective verbal skills

\section{Operational ability / Expertise / Problem Diagnosis / Solving / Crisis Management / Time Management}

Organizational commitment / Organizational loyalty / Organizational knowledge / Organizational belonging / Organizational culture

Expert human resources / Trusted human resources / Believing and religious human resources / Motivated human resources / Loyal human resources

Effective training in prevention and safety activities / Effective learning of relief activities

Reducing the pain and suffering of humanity / Increasing life expectancy / Improving public health indicators / Reducing fatalities and casualties in crises and disasters

Empathy / Compassion / Peace and Friendship / Sacrifice/ Kindness / Preservation of Human Dignity

Increasing voluntary financial aid / Increasing the participation of organizations / allocating appropriate budget

Increasing voluntary services / Increasing membership in the Society/ Increasing trust

The need to protect human life and health / The need to preserve human dignity and values / Establishing lasting cooperation and peace among human beings

Lack of attention to profitability / voluntary service/desire to serve

Impartiality in conflicts / non-participation in religious-political disputes

Lack of political and religious orientation / Prioritization based on critical issues

Maintaining independence by respecting the laws of the country / no formal or informal affiliation with other organizations

The dominant culture in society / public outlook on Red Crescent activities

Role of the senior manager in motivating human resources/perspective of the manger

Organizational laws/laws of government and governing institutions

Effective evaluation / recording feedback / material incentives / spiritual incentives 
competencies, and improvement and development of professional competencies are the four main categories of the current research and were selected as the main strategies for developing human resource competencies. In other words, in order to develop the key competencies of human resources, these competencies must be developed and improved in four dimensions, namely individual, social, professional, and organizational.

Public culture, senior management support, as well as governing rules and regulations, are three categories that influence strategies as intervening variables. Moreover, the effective and continuous evaluation of human resources competencies is an underlying category affecting the development of human resource competencies. Consequences are the output of appropriate strategies employed to develop key human resources competencies and evaluate them continuously and effectively. In the current study, the employment of appropriate strategies for evaluating and developing key human resource competencies resulted in the following consequences: human resource efficiency, provision of desirable social services, community health improvement, enhancement of human values, reinforcement of trust and public participation, and improvement of the financial capacity of the Red Crescent Society.

\section{Selective coding}

Selective coding is the process of integration and improvement of categories. At this stage of coding, a theory is formulated on the basis of the relationship among the categories in the axial coding model. It can be stated that humanity, impartiality, neutrality, independence, and voluntary service as the basic principles of the Red Crescent Society need competent human resources which can be achieved by improving and developing individual, socio-communication, professional, and organizational competencies through effective evaluation and appropriate feedback. To attain this goal, such factors as public culture, senior management support, and governing rules and regulations can act as a facilitator or hindrance.

The improvement and development of competencies enable the Red Crescent Society to attain its goals, including human resource efficiency, provision of desirable social services, improvement of community health and human values, enhancement of trust and public participation, and increasing the financial capacity of the Red Crescent Society.

\section{Describing the studied variables and questionnaire items}

To describe the variables, central and dispersion indices were used as discussed below (Table 2).

The items of the questionnaire were rated on a five-point Likert scale (1 to 5), and a number of items were set for each variable. Given that the Likert scale varied within 1-5, the numerical range of all variables must also vary in this range

Table 2. Descriptive indicators of the studied variables

\begin{tabular}{ccccc|} 
Variable & Mean & SD & Minimum & Maximum \\
Humanity & 3.175 & 1.265 & 1 & 5 \\
Voluntary service & 3.189 & 1.262 & 1 & 5 \\
Impartiality & 3.550 & 1.027 & 1 & \\
Neutrality & 3.579 & 0.976 & 1 & 5 \\
Independence & 3.304 & 1.124 & 1 & 5 \\
Public culture & 3.304 & 1.177 & 1 & 5 \\
Senior Manager Support & 3.365 & 1.030 & 1 & 5 \\
Governing rules and regulations & 3.316 & 1.233 & 1 & 5 \\
Improving and developing individual competencies & 3.402 & 1.183 & 1 & 5 \\
Improving and developing organizational competencies & 3.220 & 1.081 & 1 & 5 \\
Improving and developing communication-social competencies & 3.146 & 1.081 & 1 & 5 \\
Improving and developing professional competencies & 3.230 & 1.278 & 1 & 5 \\
Ongoing evaluation and feedback on human resource competencies & 3.655 & 0.942 & 1 & 5 \\
Human resource efficiency & 3.676 & 0.872 & 1 & 5 \\
Providing desirable social services & 3.753 & 0.948 & 1 & 5 \\
Improving community health & 3.640 & 0.976 & 1 & 5 \\
Improving human values & 3.652 & 0.938 & 1 & 5 \\
Increasing trust and public participation & 3.655 & 0.869 & 1 & 5 \\
\hline
\end{tabular}


(1-5). As presented in Table 2, all variables were scored within the range of $1-5$, signifying that the data were entered correctly.

\section{Inferential statistics}

Kolmogorov-Smirnov test: Firstly, the data normality test should be performed to use the appropriate test to assess the hypotheses. Normal distribution means that the distribution of variables is symmetric about the mean. To test the hypotheses, parametric tests are used for normally distributed data, while non-parametric tests are applied for non-normally distributed data. Kolmogorov-Smirnov test is used to test data normality. In this test, the hypotheses are as follows:

HO: The data follow a normal distribution.

H1: The data do not follow a normal distribution.

In this test, $\mathrm{P}<0.05$ indicated that our variable does not follow a normal distribution, while the $\mathrm{p}$ value higher than 0.05 is suggestive of data normality.

The results of the data normality test are displayed in Table 3. The significance level of all the studied variables in the research is calculated to be less than the chosen alpha level (0.05). Therefore, it can be concluded that the data distribution of these variables does not follow the normal statistical distribution. As a result, we should consider non-parametric methods, such as the partial least squares-structural equation modeling (PLS-SEM) method using smartpls3 software.

The Kaiser-Meyer-Olkin (KMO) index and Bartlett's test: This test assesses sample adequacy to do the factor analysis. The kmo values closer to 1 are indicative of a better sample size. Bartlett's Test of Sphericity is also used to confirm patterned relationships among the variables. Bartlett has not a value in itself; nonetheless, it is confirmed due to its Chi-Square significance, and the sphericity of the relationship is confirmed (Table 4).

In the abovementioned table, since the value of KMO statistic is $0.879(>0.7)$ and Bartlett's test is significant, it can be inferred that the adequacy of our sample size is confirmed.

\section{Structural equation modeling}

Structural models are evaluated after analyzing the measurement model. The structural model considers the relationships between the latent independent (exogenous) and dependent (endogenous) variables. The structural equation modeling only examines the hidden variables and the relationships among them. The criteria for structural modeling analysis are as follows:

1. Path coefficients (beta) and their significance (t-values)

2. Coefficient of determination (R2) endogenous latent variables

Table 3. Results of the normality of the variables

\begin{tabular}{|c|c|c|c|}
\hline Variables & $\begin{array}{l}\text { Kolmog } \\
\text { statistic }\end{array}$ & $\begin{array}{l}\text { rov-Smirnov test } \\
\text { Significance level }\end{array}$ & Test result \\
\hline Humanity & 0.117 & 0.000 & Non-normal \\
\hline Voluntary service & 0.111 & 0.000 & Non-normal \\
\hline Impartiality & 0.113 & 0.000 & Non-normal \\
\hline Neutrality & 0.100 & 0.000 & Non-normal \\
\hline Independence & 0.104 & 0.000 & Non-normal \\
\hline Public culture & 0.084 & 0.000 & Non-normal \\
\hline Senior Manager Support & 0.089 & 0.000 & Non-normal \\
\hline Governing rules and regulations & 0.091 & 0.000 & Non-normal \\
\hline Improving and developing individual competencies & 0.132 & 0.000 & Non-normal \\
\hline Improving and developing organizational competencies & 0.097 & 0.000 & Non-normal \\
\hline Improving and developing communication-social competencies & 0.112 & 0.000 & Non-normal \\
\hline Improving and developing professional competencies & 0.127 & 0.000 & Non-normal \\
\hline Ongoing evaluation and feedback on human resource competencies & 0.098 & 0.000 & Non-normal \\
\hline Human resource efficiency & 0.091 & 0.000 & Non-normal \\
\hline Providing desirable social services & 0.136 & 0.000 & Non-normal \\
\hline Improving community health & 0.108 & 0.000 & Non-normal \\
\hline Improving human values & 0.091 & 0.000 & Non-normal \\
\hline Increasing trust and public participation & 0.080 & 0.000 & Non-normal \\
\hline Increasing the financial capacity of the Society & 0.091 & 0.000 & Non-normal \\
\hline
\end{tabular}


Table 4. KMO and Bartlett's test

\begin{tabular}{|ccc|}
\hline & Indicators & Values \\
& KMO value & 0.879 \\
Bartlett's & Chi-square value & 21039.671 \\
test & Degrees of freedom & 2415 \\
& Significance level & 0.000 \\
\hline
\end{tabular}

The first criterion for examining the fit of the structural model is the significance of the $\mathrm{T}$ statistic. If the obtained value is greater than 1.96, that hypothesis is confirmed at a $95 \%$ confidence level (Table 5).

As illustrated in Table 5, the calculated tvalues between all independent and dependent variables in the model are>1.96 and are significant at a $95 \%$ confidence level.

\section{Goodness of Fit Indicators for the tested Model}

The relationships between observant and latent variables are considered and measured in a measurement model. All the tests related to the measurement model in PLS software are reviewed in this section. The following criteria were used to evaluate the fit of measurement models:

1. Reliability test (Cronbach's alpha and composite reliability (CR) coefficient)

2. Convergent validity (significance of factor loadings, homogeneity, average variance extracted (AVE), and comparison between CR and AVE

3. Reliability tests (Cronbach's alpha and composite reliability coefficient)

According to the data analytic algorithm in PLS, after measuring the factor loadings of the indicators, Cronbach's alpha and composite reliability coefficient need to be calculated and reported. According to the definition of Cronbach's alpha, the more the items are positively correlated, the higher the alpha coefficient. On the other hand, if the items are less strongly associated, the alpha coefficient will be lower. It is evident that the closer Cronbach's alpha coefficient is to 1.0 the greater the internal consistency of the items, and consequently, the more homogeneous the items. Based on Cronbach's alpha, reliability coefficients of $0.45,0.7$, and 0.95 were regarded as low, moderate and acceptable, and high, respectively. Since this index is very strict, a more modern criterion called Composite Reliability (CR) is used to check the internal consistency of the measurement model in the PLS method. It can be calculated using the following formula:

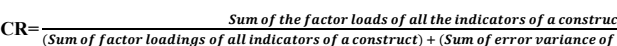

The optimum CR value in exploratory research varies from $0.6-0.7$, while it is between 0.7 and 0.9 in more advanced research. The results of these two criteria are shown in the Table 6.

As displayed in Table 6, Cronbach's alpha coefficients and the composite reliability are acceptable for all research constructs.

\section{Average mean extracted (AVE) and its comparison with composite reliability coefficient}

In PLS modeling, another suitable criterion for evaluating the measurement model is that the construct should have the most common variance with its marker variables. The last confirmatory criterion for convergent validity is the comparison between the composite reliability and average mean extracted. To confirm convergence validity, $\mathrm{CR}$ must be higher than AVE (Table 7).

The appropriate value of AVE is 0.5. As presented in Table 7, AVE was > 0.5 in all variables; therefore, the convergent validity was confirmed using this index. Moreover, CR was >AVE in all latent variables, and the fourth required condition of convergent validity was met. According to the performed tests, it can be concluded that the research model has good convergence validity.

Coefficient of determination (R2) of the endogenous latent variables: It is the second

Table 5. Coefficients standardized factor loadings and t-value among the latent variables

\begin{tabular}{|cccc|}
\hline Independent variables & Dependent variable & Path coefficient ( $\boldsymbol{\beta}) \boldsymbol{\beta}$ & t-value \\
\hline Causal conditions & Competency development & 0.238 & 3.437 \\
Intervening variable & Competency development & 0.251 & 4.040 \\
Competency development & Consequences & 0.737 & 18.211 \\
Causal conditions*Intervening variable & Competency development & 0.071 & 1.193 \\
\hline
\end{tabular}

A t-value $>1.96$ confirms the relationships. 
Table 6. Results of Cronbach's alpha and composite reliability coefficient

\begin{tabular}{ccc} 
Variable & (Alpha >0. 7) & (CR>0. 7) \\
Providing desirable social services & 0.875 & 0.941 \\
Improving human values & 0.917 & 0.938 \\
Ongoing evaluation and feedback on human resource competencies & 0.743 & 0.833 \\
Independence & 0.801 & 0.909 \\
Humanity & 0.779 & 0.871 \\
Impartiality & 0.796 & 0.907 \\
Neutrality & 0.727 & 0.880 \\
Voluntary service & 0.875 & 0.923 \\
Increasing trust and public participation & 0.77 & 0.872 \\
Increasing the financial capacity of the population & 0.846 & 0.907 \\
Improving and developing communication-social competencies & 0.852 & 0.901 \\
Improving and developing professional competencies & 0.877 & 0.915 \\
Improving and developing organizational competencies & 0.780 & 0.901 \\
Improving and developing individual competencies & 0.776 & 0.849 \\
Improving community health & 0.731 & 0.881 \\
Senior Management Support & 0.734 & 0.883 \\
Public culture & 0.793 & 0.830 \\
Governing rules and regulations & 0.888 & 0.916 \\
Human resources efficiency & 0.831 & 0.883 \\
\hline
\end{tabular}

criterion for assessing the structural model, indicating the effect of an exogenous variable on an endogenous variable. The three values of $0.19,0.33$, and 0.67 are regarded as weak, moderate, and high coefficients of determination. The higher $R^{2}$ of the endogenous variables demonstrates the better fit of the model. According to Hensler et al. (2009), if in a model, an endogenous construct is affected by one or two exogenous constructs, the $R^{2} \geq 0.33$ indicates the strength of the relationship between that construct and endogenous constructs. The presence of more independent variables increases $R^{2}$. Therefore, a higher $R^{2}$ is required for model fitting when the number of independent variables which explain a dependent variable is higher (Table 8).

The $R^{2}$ value for the endogenous variables of the model was calculated to be 0.377 and 0.544 , respectively.

Table 7. Results of assessing convergent validity based on AVE criterion

\begin{tabular}{ccccc}
\hline Variable & A VE & CR & CR>A VE \\
Provision of desirable social services & 0.889 & 0.941 & OK \\
Improving human values & 0.751 & 0.938 & OK \\
Ongoing evaluation and feedback on human resource competencies & 0.556 & 0.833 & OK \\
Independence & 0.834 & 0.909 & OK \\
Humanity & 0.694 & 0.871 & OK \\
Impartiality & 0.830 & 0.907 & OK \\
Neutrality & 0.785 & 0.880 & OK \\
Voluntary service & 0.801 & 0.923 & OK \\
Increasing trust and public participation & 0.694 & 0.872 & OK \\
Increasing the financial capacity of the population & 0.766 & 0.907 & OK \\
Improving and developing communication-social competencies & 0.694 & 0.901 & OK \\
Improving and developing organizational competencies & 0.730 & 0.915 & OK \\
Improving and developing individual competencies & 0.820 & 0.901 & OK \\
Improving community health & 0.532 & 0.849 & OK \\
Senior Management Support & 0.788 & 0.881 & OK \\
Public culture & 0.790 & 0.883 & OK \\
Governing rules and regulations & 0.620 & 0.830 & OK \\
Human resources efficiency & 0.647 & 0.916 & OK \\
\hline
\end{tabular}




\section{Assessment of general model (Goodness of fit (GOF) index)}

After evaluating the measurement and structural models, the general model (sum of measurement and structural models) was also assessed. For this purpose, Tenenhaus et al. (2004) introduced the GOF index. This index is calculated by GOF $=\sqrt{\overline{\text { Communalltles }} \times \overline{R^{2}}}$ formula, and three values of $0.01,0.25$, and 0.36 are considered weak, medium, and strong values. In fact, when this value is closer to 1 , the general model is more strongly validated. This index is the mean squared of the mean shared values (communality) and the mean coefficient of determining (R Square Average).

$$
\text { GOF }=\sqrt{\overline{\text { Communalitles }} \times \overline{R^{2}}} \text { CommunalitY }
$$

As displayed in Table 9, the GOF standard value was $0.649(>0.36)$, suggesting the strong fit of the overall model of the research (Table 9).

Table 8. Coefficient of determination of dependent variables

\begin{tabular}{|ccc|}
\hline Dependent variables & $\boldsymbol{R}^{2}$ & Description \\
\hline Competency development & 0.377 & Three values of $0.19,0.33$, and 0.67 are \\
Consequences & 0.544 & regarded as weak, medium, and strong $\mathrm{R}^{2}$ \\
\hline
\end{tabular}

Table 9. Results of general fitting of the model using GOF criterion

\begin{tabular}{|c|cc|c|}
\hline Variable & Communality & $\boldsymbol{R}^{2}$ & GOF \\
\hline Competency development & 0.915 & 0.377 & $\sqrt{0.915 \times 0.461}=0.649$ \\
\hline Consequences & 0.916 & 0.544 & \\
\hline
\end{tabular}

\section{Discussion and Conclusion}

Most of the interviewees highlighted the importance of developing human resources competencies and asserted that this competency makes an organization superior to its counterparts in the industry and provides the ground for organizational growth and development. The competency-based approach to employees, as a strategic principle, is in fact the link between employees and their organization. Therefore, organizations can compete with their counterparts around the world relying on the competencies of employees.

Therefore, due to the crucial importance of human resources competencies, special attention should be devoted to this critical issue in organizations. The Red Crescent Society of the Islamic Republic of Iran is no exception in this regard. So far, no activity has been performed on this field by Red Crescent Society as a relief and non-profit organization. Nevertheless, most activities performed in this society are focused on preparedness and first aid training. Moreover, under the slogan "ready family in disasters", numerous activities in this organization are directed toward public relief education.

Furthermore, the Red Crescent Society, as the custodian of relief and rescue, is responsible for the management of disaster victims. This issue is of increasing importance due to science and technology developments, rapid technological advancement, and the growing population of the world. Therefore, the competencies of the Red Crescent aid workers should be promoted so that they can effectively perform their activities in an organization which plays a peculiar role in providing relief to community members in the time of crises.

The improvement and development of individual competencies, improvement and development of organizational competencies, improvement and development of social competencies, and improvement and development of professional competencies are the four main categories of the current research and were selected as the main strategies for developing human resource competencies. In other words, in order to develop the key competencies of human resources, these competencies must be developed and improved in four dimensions, namely individual, social, professional, and organizational.

Public culture, senior management support, as well as governing rules and regulations, are three categories that influence strategies as intervening variables and affect the effective and continuous evaluation and feedback on human resource competencies as an underlying category of human resource competency development. Consequences are the output of employed appropriate strategies 
to develop key human resource competencies and evaluate them continuously and effectively. In the current study, the employment of appropriate strategies for evaluating and developing key human resource competencies resulted in the following consequences: human resource efficiency, provision of desirable social services, community health improvement, enhancement of human values, reinforcement of trust and public participation, and improvement of the financial capacity of the Red Crescent Society.

In order to increase the competence of their employees, the Red Crescent Society as a humanitarian relief organization should devote assiduous attention to such principles as humanity, impartiality, neutrality, independence, and voluntary service. For instance, the principle of humanity among the Red Crescent staff causes them to take spontaneous steps to carry out their activities on their own and voluntarily pursue their missions. Based on the results of PLS structural equation modeling, there was a positive and significant relationship between the principle of humanity and the development of competencies among the Red Crescent staff.

The principle of impartiality enables the employees to provide disaster victims with care and support without any national, racial, political, or religious orientations. Furthermore, the results of PLS structural equitation modeling showed that competency development was positively and significantly correlated with impartiality, neutrality, and independence. Public culture, senior management support, as well as governing rules and regulations, are among the factors influencing the development of Red Crescent staff competencies since the prevailing general culture in an organization can help to expand and improve employees' competencies.

Senior management support in organizations provides the basis for competency development since this support can help employees improve their competencies. If they focus on the competencies and skills of employees, organizational rules and regulations can pave the ground for meritocracy and highlighting the development of employee competencies. The managers of organizations know that they must take advantage of employees' competencies in order to respond to today's changing world; moreover, the new organizations cannot be managed as before.

The results of the quantitative analysis also confirmed the positive and significant relationship of competency development with public culture, senior management support, and governing rules and regulations. However, it should be considered that all these conditions must be met through the assessment and development of competencies. This development can be accomplished by the improvement and development of individual, organizational, communication-social, and professional competencies. The mere attention to individual competencies cannot promote organizations; rather special attention must be devoted to the development of organizational and communication-social competencies in society and in relation to other organizations. Paying attention to social skills and competencies used to communicate with other organizations can give rise to cooperation, growth, and promotion of the organization itself.

The results of the quantitative analysis also pointed to the positive and significant relationship between each of these strategies and competency development and evaluation. This signifies that these strategies can be regarded as effective steps towards competency development and assessment among the Red Crescent staff. Finally, it is worth noting that highlighting competency development and evaluation can bring many consequences for the organization, such as human resources efficiency and provision of desirable social services, which in turn, promotes community health. Furthermore, the improvement of human resources competencies in the Red Crescent employees leads to increased cooperation of employees. In so doing, they can improve the living standards of people with more trust and participation and promote human values in the community.

\section{Acknowledgments}

The authors' deepest appreciation goes to the Vice Chancellors for Education, Research and Technology, Deputy of Human Resources Development of the Red Crescent Society, as well as Iran-Helal Applied-Science Higher Education Institute for their cooperation and support in carrying out this dissertation.

\section{Conflict of Interests}

The authors have no conflict of interest to declare. 


\section{References}

1. Lee FH, Lee TZ, Wu WY. The relationship between human resource management practices, business strategy and firm performance: evidence from steel industry in Taiwan. Int J Hum Resource Manag 2010; 21(9): 1351-72.

2. Dubois DD, Rothwell WJ. Competency-based human resource management: Discover a new system for unleashing the productive power of exemplary performers. Boston: Nicholas Brealey; 2004.

3. Campion MA, Fink AA, Ruggeberg BJ, Carr L, Phillips GM, Odman RB. Doing competencies well: best practices in competency modeling. Person Psychol 2011; 64(1): 225-62.

4. Judrups J, Zandbergs U, Kazakovs M. Competence based human resource development solution. Eng Rural Dev 2015; 14: 669-74.
5. Bohlouli M, Ansari F, Kakarontzas G, Angelis L. An adaptive model for competences assessment of IT professionals. Integrated systems: innovations and applications. Cham: Springer; 2015 (In Persian)

6. Hajikarimi A, Rezaeian A, Hadizadeh A, Bonyadi NA. Designing the competencies model of human resource managers in the public sector of Iran. J Pub Admin Perspective 2012; 2(8): 23-43 (In Persian)

7. Ahmadi SA, Darvish H, Sobhanifar MJ, Fazeli Kebria H. Designing, and explaining conceptual model of human resources competencies in Nahj alBalagha by utilizing content analysis method and ISM. Basij Strategic Stud Quart 2015; 17(64): 27 (In Persian)

8. Narenji Sani F, Rouhani S, Sharifi M, Jamaledini $\mathrm{SH}$. Developing the strategies of educational management in Iranian Red Crescent. J Train Dev Hum Resour 2017; 4(4): 97-114. (In Persian) 